\title{
TOPOLOGICAL ANALYSIS AND FREQUENCY DEPENDENT HYPERPOLARIZABILITY CALCULATIONS OF FDDNP: A DFT STUDY
}

\author{
Keivan Akhtari ${ }^{\mathrm{a}^{*}}$, Keyumars Hassanzadeh ${ }^{\mathrm{b}}$, Bahareh Fakhraei ${ }^{\mathrm{a}}$, Ghazal Akhtari ${ }^{\mathrm{a}}$ \\ ${ }^{a}$ Department of Physics, University of Kurdistan, P.O.Box 416, Sanandaj, Iran \\ ${ }^{b}$ Young Researchers and Elites Club, Islamic Azad University, Sanandaj, Iran \\ *e-mail: k1akhtari@yahoo.com; phone: (+98) 8733289430; fax: (+98) 8733247713
}

\begin{abstract}
The topological and first-hyperpolarizability properties of 2-(1-\{6-[(2-fluoroethyl)(methyl)amino]-2naphthyl \} ethylidene)malononitrile (FDDNP) were studied using DFTB3LYP method. The static and dynamic electronic (hyper)polarizabilities of conformers were calculated and a simple two-state model was employed to explain the first hyperpolarizability differences in two conformers. The second harmonic generation property was evaluated at the typical wavelengths of Cr:forsterite, Nd:YAG(neodymium-doped yttrium aluminium garnet) and Ti:sapphire lasers to predict the compound conformers potency for second harmonic generation imaging in biological studies.
\end{abstract}

Keywords: second-harmonic generation, FDDNP, near-infrared, QTAIM.

Received: August 2016/ Revised final: October 2016/ Accepted: October 2016

Introduction

Optical imaging techniques based on nonlinear optical phenomena have been widely incorporated in microscopy using ultra-fast pulsed lasers. There have been described many applications in cellular and tissue imaging due to the ability to spatially resolve subcellular details with high molecular contrast [1].

The employed near-infrared (NIR) wavelengths reduce scattering and maximize tissue penetration. These characteristics boost nonlinear microscopy as an elective method for imaging cells with micrometer-resolution deep into the living tissues. Both labeled and label-free second harmonic generation (SHG) imaging techniques have been employed in biological studies. For example, the non-centrosymmetric structure of fibrillar collagen is the major source of the SHG signals in different tissues [2,3], and for cellular and subcellular samples. For this purpose, several SHG labels have been used $[4,5]$.

The 2-(1-\{6-[(2-fluoroethyl)(methyl)amino]-2-naphthyl\}ethylidene)malononitrile (FDDNP) is a functional fluorescent biomarker belonging to the family of dicyanovinyl naphthalene labels, which binds to its ligand through hydrophobic interactions [6]. FDDNP also binds to amyloid plaques with high affinity [7]. Fluorescence imaging microscopy of amyloid aggregates has a key role in diagnosing Alzheimer's disease and other neurodegenerative diseases $[8,9]$.

According to the importance of second-harmonic generation, as one of the nonlinear excitation families with deep imaging, 3D capabilities and intrinsic contrast in certain tissues, the study of nonlinear optical properties of biomaterials and their labels plays an essential role in development of medicinal optic devices. So far, only a few electronic structure studies on FDDNP and other dicyanovinyl naphthalene biomarkers, based on $a b$ initio calculations have been performed. Petrič et al. [6] performed a combined experimental and theoretical study on FDDNP and its analogs to explain the chemical modification effects on binding affinity of biomarkers to amyloid plaques and their fluorescence Quantum yields. Given the close relationship between the structure and nonlinear activities, and the functional importance of default molecule, it is necessary to develop a deeper vision on electron-topological properties, in order to predict its different optical capabilities. In the present work we consider both structural and nonlinear optical properties of FDDNP to introduce it as a potent material in nonlinear optical imaging of biological samples.

Thereupon in this work, beside the study of conformational effect on structural properties, the static and frequency-dependent electronic (hyper)polarizabilities of the FDDNP conformers were also computed. For this purposes, the nonlinear optical properties related to the excited-state dipole moment were studied using a simple two-state model (TSM) [10].

\section{Computational details}

The geometry optimization, frequency and single point energy calculations were carried out for all structures, using the Density Functional Theory method supplied in the GAUSSIAN98W suite of programs [11].

The B3LYP functional [12-14] with the 6-31G(d,p) basis set [15] were used.

The Time-Dependent Density Functional Theory (TD-DFT) was used to calculate the excited state energies and excited state dipole moments. The 10 lowest excited states were considered. The orbital transition contributions were obtained using GAUSSSUM 2.2 program [16]. 
To estimate the solvent effect on the studied optical parameters, polarizable continuum model (PCM) calculations were performed [17]. The topological analyses were carried out using Multiwfn program [18].

\section{Results and discussion}

\section{Geometry and Natural Bond Orbital (NBO) studies}

Two stable conformations of the FDDNP can theoretically exist [6]. The optimized structures are shown in Figure 1. The C2-C6-C9-C10 dihedral angles for two conformers are approximately $31^{\circ}$ (Syn form) and $142^{\circ}$ (Anti form). The Syn conformer has lower total energy than the Anti form by $4.98 \mathrm{~kJ} / \mathrm{mol}$.

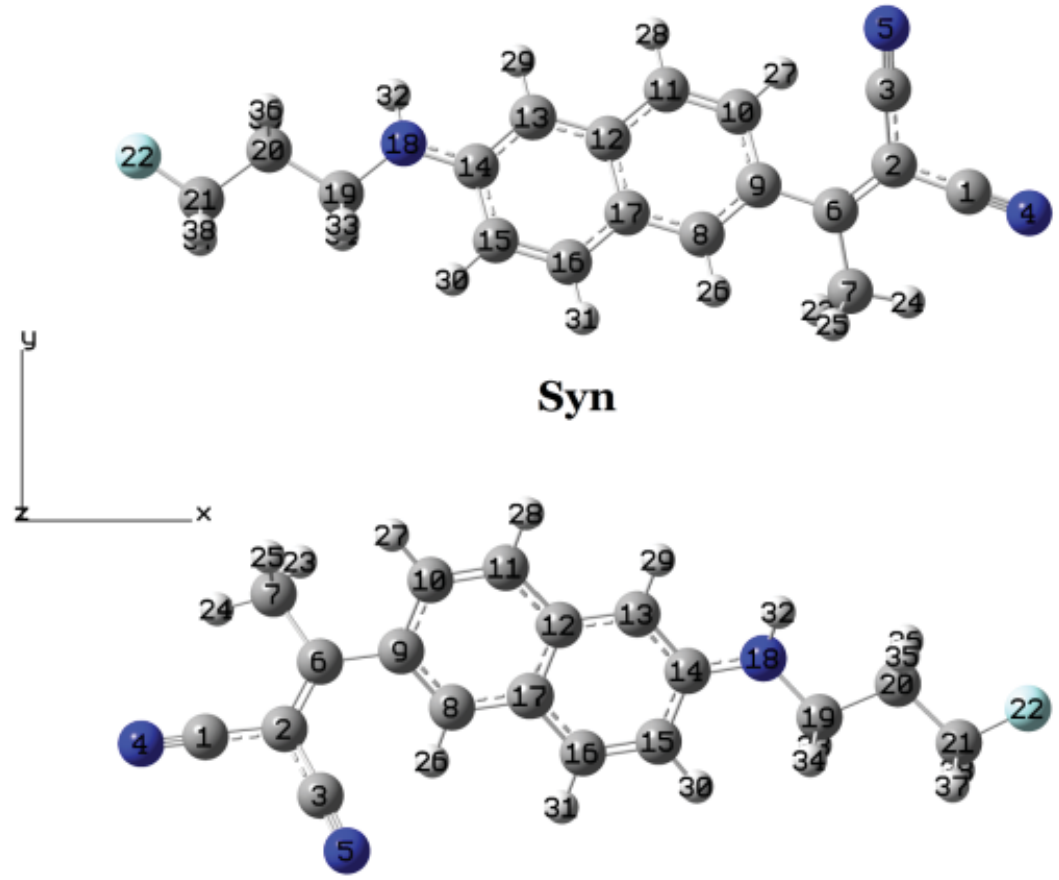

Anti

Figure 1. Structures of two possible minimum energy conformations of FDDNP.

Intra-molecular hyperconjugative interactions can be used as a measure of the interaction intensity between electron-acceptor orbitals. In fact, the larger the value of the hyperconjugative interaction, the more intensive the interaction between the donor and acceptor, and the greater the extent of conjugation of the whole system is [19].

The role of hyperconjugative interactions in the stabilization of Syn and Anti conformers has been studied using NBO analysis. For a better comparison of the conformers' stability, the same values of the donor-acceptor interactions with large differences $(\geq 0.4 \mathrm{~kJ} / \mathrm{mol})$ have been summarized in Table 1 . Upon the data, hyperconjugation interactions are more favored for the Syn form. The interactions such as $\pi \mathrm{C} 8-\mathrm{C} 9 \rightarrow \pi^{*} \mathrm{C} 2-\mathrm{C} 6$ and $\pi \mathrm{C} 2-\mathrm{C} 6 \rightarrow \pi^{*} \mathrm{C} 8-\mathrm{C} 9$ play an important role in conformational properties of FDDNP. In addition, the sum values of hyperconjugation interactions are in accordance with the total energy values.

Table 1

Important hyperconjugative interactions $\left(\mathrm{kJ} \mathrm{mol}^{-1}\right)$ for FDDNP conformers.

\begin{tabular}{lccc}
\hline Donor & Acceptor & Syn & Anti \\
\hline$\pi \mathrm{C} 2-\mathrm{C} 6 \rightarrow$ & $\pi^{*} \mathrm{C} 1-\mathrm{N} 4$ & 85.19 & 85.94 \\
& $\pi^{*} \mathrm{C} 3-\mathrm{N} 5$ & 84.10 & 84.77 \\
$\sigma \mathrm{C} 6-\mathrm{C} 9 \rightarrow$ & $\pi^{*} \mathrm{C} 8-\mathrm{C} 9$ & 30.92 & 27.74 \\
& $\sigma^{*} \mathrm{C} 8-\mathrm{C} 9$ & 11.25 & 12.47 \\
$\sigma \mathrm{C} 7-\mathrm{H} 23 \rightarrow$ & $\sigma^{*} \mathrm{C} 9-\mathrm{C} 10$ & 9.71 & 8.79 \\
$\sigma \mathrm{C} 8-\mathrm{C} 9 \rightarrow$ & $\sigma^{*} \mathrm{C} 2-\mathrm{C} 6$ & 10.17 & 10.63 \\
& $\sigma^{*} \mathrm{C} 6-\mathrm{C} 9$ & 12.05 & 12.84 \\
$\pi \mathrm{C} 8-\mathrm{C} 9 \rightarrow$ & $\sigma^{*} \mathrm{C} 9-\mathrm{C} 10$ & 14.43 & 14.94 \\
& $\pi^{*} \mathrm{C} 2-\mathrm{C} 6$ & 75.81 & 71.88 \\
& $\pi^{*} \mathrm{C} 10-\mathrm{C} 11$ & 69.08 & 72.01 \\
\hline
\end{tabular}


Continuation of Table 1

\begin{tabular}{lccc}
\hline Donor & Acceptor & Syn & Anti \\
\hline$\sigma \mathrm{C} 8-\mathrm{C} 17 \rightarrow$ & $\sigma^{*} \mathrm{C} 6-\mathrm{C} 9$ & 13.81 & 14.89 \\
$\sigma \mathrm{C} 8-\mathrm{H} 26 \rightarrow$ & $\sigma^{*} \mathrm{C} 8-\mathrm{C} 9$ & 4.81 & 5.23 \\
& $\sigma^{*} \mathrm{C} 9-\mathrm{C} 10$ & 17.07 & 17.99 \\
& $\sigma^{*} \mathrm{C} 12-\mathrm{C} 17$ & 18.83 & 19.25 \\
$\sigma \mathrm{C} 9-\mathrm{C} 10 \rightarrow$ & $\sigma^{*} \mathrm{C} 6-\mathrm{C} 9$ & 10.50 & 9.92 \\
$\pi \mathrm{C} 10-\mathrm{C} 11 \rightarrow$ & $\pi^{*} \mathrm{C} 8-\mathrm{C} 9$ & 63.47 & 60.50 \\
& $\pi^{*} \mathrm{C} 12-\mathrm{C} 17$ & 65.19 & 14.68 \\
$\sigma \mathrm{C} 10-\mathrm{H} 27 \rightarrow$ & $\sigma^{*} \mathrm{C} 8-\mathrm{C} 9$ & 68.07 & 5.23 \\
& $\sigma^{*} \mathrm{C} 10-\mathrm{C} 11$ & 15.27 & 18.16 \\
$\sigma \mathrm{C} 11-\mathrm{C} 12 \rightarrow$ & $\sigma^{*} \mathrm{C} 11-\mathrm{C} 12$ & 5.69 & 18.62 \\
\hline $\mathrm{Sum}$ & $\sigma^{*} \mathrm{C} 10-\mathrm{H} 27$ & 9.50 & 704.09 \\
\hline
\end{tabular}

\section{Topological analysis}

The topological study of electron density based on Quantum Theory of Atom In Molecules (QTAIM) as a rigorous interpretative method can unambiguously describe atoms existing in the system. In this theory the chemical bonds can be defined as the set of lines known as bond paths (BPs) linking neighboring atoms. In addition, other concepts and quantities are also necessary to characterize a bonding interaction, namely critical point (CP), electron density $\rho(r)$, Laplacian of electron density $\nabla^{2} \rho(r)$, kinetic energy density $[G(r)]$, potential energy density $[V(r)]$, and total energy density $[H(r)][20]$.

Hydrogen bonding can be explained and classified as a main factor in determining the preferred conformers according to the Roza's criteria [21]:

1. weak hydrogen bonds: $\nabla^{2} \rho_{\mathrm{BCP}}>0$ and $H_{\mathrm{BCP}}>0$;

2. medium hydrogen bonds: $\nabla^{2} \rho_{\mathrm{BCP}}>0$ and $H_{\mathrm{BCP}}<0$;

3. strong hydrogen bonds: $\nabla^{2} \rho_{\mathrm{BCP}}<0$ and $H_{\mathrm{BCP}}<0$,

where $\nabla^{2} \rho_{\mathrm{BCP}}$ and $H_{\mathrm{BCP}}$ are the Laplacian of electron density and the total electron energy density at critical point respectively.

The nature of hydrogen bonds can be specified using the following criteria [22]:

1. for $\frac{G_{B C P}}{\left|V_{B C P}\right|}>1$, the hydrogen bond has non-covalent nature;

2. for $0.5<\frac{G_{B C P}}{\left|V_{B C P}\right|}<1$, the hydrogen bond is partly covalent.

At the first step, we verified if $\mathrm{C} 3 \cdots \mathrm{H} 27$ and $\mathrm{C} 3 \cdots \mathrm{H} 26$ (see Figure 2) were in fact hydrogen bonds. Then, the strength of bonds was studied through the criteria above. Koch and Popelier [23] established some criteria for hydrogen atom that are involved in hydrogen bond as follows:

1. increasing the net positive charge $[q(H)]$;

2. decreasing the first atomic dipole moment $[M(H)]$;

3. decreasing the atomic volume $[V(H)]$;

4. increasing the atomic total energy $[E(H)]$.

To compare the atomic properties with a reference, the C2-C6-C9-C10 dihedral angle was fixed at $90^{\circ}$ and QTAIM calculations were performed to obtain the atomic properties of H26 and H27 when they are not involved in hydrogen bond. The atomic QTAIM parameters related to H26 and H27 for Syn conformer, Anti conformer and the reference state are gathered in Table 2 . As can be seen, all Koch's criteria for identification of C3 $\cdots \mathrm{H} 26$ and $\mathrm{C} 3 \cdots \mathrm{H} 27$ as hydrogen bonds are satisfied.

Table 2

Atomic properties of $\mathrm{H26}$ and $\mathrm{H} 27$ obtained by QTAIM for different dihedral angles.

\begin{tabular}{lccccc}
\hline $\begin{array}{c}\text { C2-C6-C9-C10 } \\
\text { dihedral angle }\end{array}$ & $\begin{array}{c}\text { Hydrogen atom } \\
\text { number }\end{array}$ & $q(H)$ & $M(H)$ & $V(H)$ & $E(H)$ \\
\hline $31^{\circ}($ Syn $)$ & 27 & 0.029917 & 0.141198 & 43.083 & -4.940696 \\
$142^{\circ}$ (Anti) & 26 & 0.027665 & 0.137417 & 43.477 & -4.938453 \\
$90^{\circ}$ (Reference) & 27 & 0.004345 & 0.151833 & 49.105 & -4.960837 \\
& 26 & 0.000881 & 0.146968 & 49.480 & -4.960837 \\
\hline
\end{tabular}




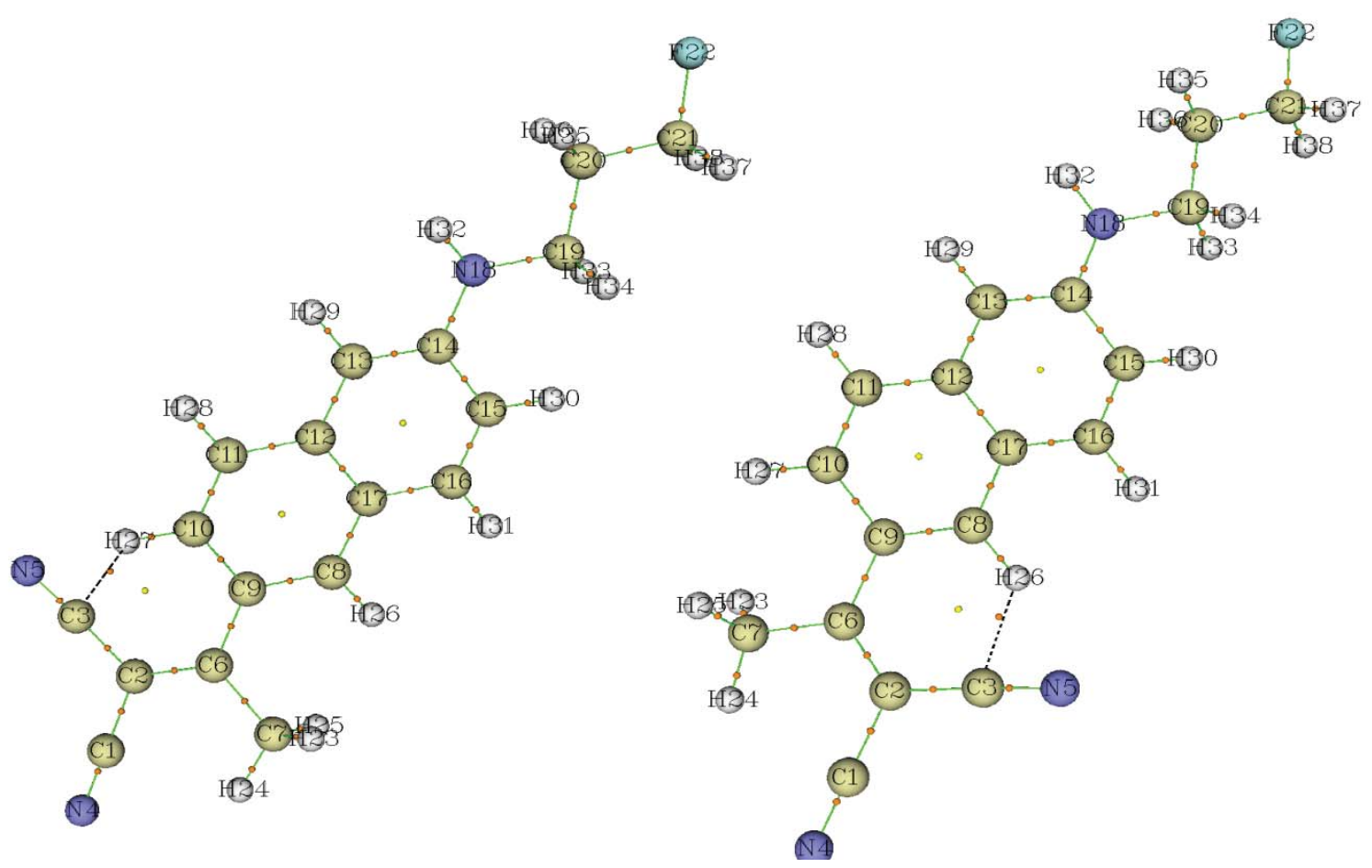

(a)

(b)

Figure 2. Molecular graphs for the electron density topology of (a) Syn and (b) Anti forms, with their bond critical points (BCP) and ring critical points (RCP).

For classification of the intra-molecular hydrogen bonds in Syn and Anti conformers, the topological quantities at characteristic critical points (see Figure 2) have been calculated and summarized in Table 3. Both Laplacians of electron density at the bond critical points are positive, which reveals a depletion of electron charge in these bond critical points.

Table 3

Topological properties of FDDNP conformers.

\begin{tabular}{cccccccccr}
\hline Conformer & $\begin{array}{c}\text { Critical } \\
\text { points }\end{array}$ & $\begin{array}{c}\text { Atoms } \\
\text { involved }\end{array}$ & $\rho(r)$ & $\nabla^{2} \rho(r)$ & $G(r)$ & $V(r)$ & & $H(r)$ & $\varepsilon^{a}$ \\
\hline \multirow{5}{*}{ Syn } & BCP & C3, H27 & 0.01249 & 0.04874 & 0.00972 & -0.00725 & 1.3400 & 0.00247 & 0.28697 \\
& RCP & $\begin{array}{c}\text { C2, C3, } \\
\text { C6, C9, C10, }\end{array}$ & 0.00995 & 0.05177 & 0.00976 & -0.00659 & 1.4824 & 0.00318 & -1.33429 \\
& \multicolumn{7}{c}{ H27 } \\
Anti & BCP & C3, H26 & 0.01222 & 0.04780 & 0.00949 & -0.00703 & 1.3504 & 0.00246 & 0.34337 \\
& RCP & C2, C3,C6, & 0.01019 & 0.05173 & 0.00979 & -0.00665 & 1.4717 & 0.00314 & -1.35175 \\
& C8, C9, H26 & & & & & & & \\
\hline
\end{tabular}

${ }^{a}$ Ellipticity (c) is another topological parameter that measures the bond's stability. The high value of ellipticity indicates the lower bonds stability [22].

In addition, the ratio of related to critical points of $\mathrm{C} 3 \cdots \mathrm{H} 27$ and $\mathrm{C} 3 \cdots \mathrm{H} 26$ are 1.34 and 1.35 respectively. Then according to Roza's criterion, both intra-molecular bonds can be classified as weak hydrogen bonds. Figure 2 shows that the formation of intra-molecular hydrogen bonds leads to a ring critical point (RCP) in each conformer.

\section{Frequency dependence of linear and nonlinear optical properties}

In order to investigate the linear and nonlinear optical performances of FDDNP conformers, polarizability $(\alpha)$, polarizability anisotropy $(\Delta \alpha)$ and first hyperpolarizability $(\beta)$ have been calculated according to the following relations [24-26]: 


$$
\begin{aligned}
& \alpha=1 / 3\left(\alpha_{x x}+\alpha_{y y}+\alpha_{z z}\right), \\
& \Delta \alpha=2^{-1 / 2}\left[\left(\alpha_{x x}-\alpha_{y y}\right)^{2}+\left(\alpha_{x x}-\alpha_{z z}\right)^{2}+\left(\alpha_{y y}-\alpha_{z z}\right)^{2}+6\left(\alpha_{x y}{ }^{2}+\alpha_{x z}{ }^{2}+\alpha_{y z}{ }^{2}\right)\right]^{1 / 2},
\end{aligned}
$$

and

$$
\beta=\left(\beta_{x}^{2}+\beta_{y}^{2}+\beta_{z}^{2}\right)^{1 / 2}
$$

where

$\beta_{x}=\beta_{x x x}+\beta_{x y y}+\beta_{x z z}$

$\beta_{y}=\beta_{y y y}+\beta_{y z z}+\beta_{x x y}$

$\beta_{z}=\beta_{x x z}+\beta_{y y z}+\beta_{z z z}$

Polarizability and hyperpolarizability parameters for some common frequencies in functional lasers are gathered in Table 4. As can be seen, the average polarizability, polarizability anisotropy, and Electro-Optic Pockels Effect (EOPE)-hyperpolarizability are increased with increasing frequency. SHG-hyperpolarizability has maximum value in $\omega=0.051770 \mathrm{au}$, which is the typical wavelength of the Ti:sapphire laser $(880 \mathrm{~nm})$

The SHG hyperpolarizability $(\beta(-2 \omega, \omega, \omega))$, EOPE-hyperpolarizability $(\beta(-\omega, \omega, 0))$ esu $\left(\times \mathbf{1 0}^{-33}\right)$,

Table 4

\begin{tabular}{|c|c|c|c|}
\hline $\begin{array}{l}\text { Linear and nonlinear } \\
\text { optical parameters }\end{array}$ & Syn & Anti & Frequency \\
\hline$\beta(-2 \omega, \omega, \omega)$ & 81208.67 & 77573.81 & \\
\hline$(\beta(-\omega, \omega, 0))$ & 81160.21 & 77564.57 & $\omega=0.000000$ \\
\hline$\alpha(-\omega, \omega)$ & 37.90 & 37.14 & \\
\hline$\Delta \alpha(-\omega, \omega)$ & 48.72 & 44.83 & \\
\hline$\beta(-2 \omega, \omega, \omega)$ & 180936.86 & 170314.51 & \\
\hline$(\beta(-\omega, \omega, 0))$ & 102024.86 & 97054.33 & $\omega=0.037040$ \\
\hline$\alpha(-\omega, \omega)$ & 39.18 & 38.32 & Cr:forsterite \\
\hline$\Delta \alpha(-\omega, \omega)$ & 51.98 & 47.68 & \\
\hline$\beta(-2 \omega, \omega, \omega)$ & 271510.12 & 254866.48 & \\
\hline$(\beta(-\omega, \omega, 0))$ & 111013.40 & 1094367.44 & $\omega=0.042820$ \\
\hline$\alpha(-\omega, \omega)$ & 39.67 & 38.78 & Nd:YAG \\
\hline$\Delta \alpha(-\omega, \omega)$ & 53.19 & 48.80 & \\
\hline$\beta(-2 \omega, \omega, \omega)$ & 1165261.93 & 1126879.80 & \\
\hline$(\beta(-\omega, \omega, 0))$ & 130949.53 & 124041.44 & $\omega=0.051770$ \\
\hline$\alpha(-\omega, \omega)$ & 40.65 & 39.69 & Ti:sapphire \\
\hline$\Delta \alpha(-\omega, \omega)$ & 55.82 & 51.08 & \\
\hline$\beta(-2 \omega, \omega, \omega)$ & 699461.39 & 585507.27 & \\
\hline$(\beta(-\omega, \omega, 0))$ & 162155.32 & 150656.64 & $\omega=0.059950$ \\
\hline$\alpha(-\omega, \omega)$ & 41.89 & 40.84 & Ti:sapphire \\
\hline$\Delta \alpha(-\omega, \omega)$ & 59.10 & 53.98 & \\
\hline
\end{tabular}
average polarizability $(\alpha(-\omega, \omega))$, and polarizability anisotropy $(\Delta \alpha(-\omega, \omega))$ esu $\left(\times 10^{-24}\right)$ calculated at B3LYP/6-31G(d,p) level of theory.

$N d: Y A G$ is the neodymium-doped yttrium aluminium garnet.

Compared to other lasers, Ti:sapphire laser is at an advantage in terms of transparency considerations of melanin, hemoglobin and water when operating at near-IR wavelengths, and short pulse duration ( $100 \mathrm{fs})$ of this laser. In such short duration, only a few nanojoules of energy transfers to samples [27,28]. Therefore, employing FDDNP label for biological microscopy, in addition to high performance in second harmonic generation, protects cells and tissues from photodamaging.

$p$-Nitroaniline (pNA) is prototypical compound used in the study of the nonlinear optical (NLO) properties as a reference one. Hence, we calculated SHG-hyperpolarizability of this molecule at $\lambda=880 \mathrm{~nm}$. The calculated value is 43.99 times smaller than the same value of FDDNP (Syn form). Thus, we can conclude that the FDDNP has considerable nonlinear optical properties. 
To study the medium effect on the optical properties of the FDDNP conformers, the water, DMSO, and benzene solvent were employed to simulate the protic polar, aprotic polar and nonpolar media. Similar method has been adopted to calculate thermodynamic and electronic properties in biological environments [29-31]. The linear and nonlinear optical parameters of FDDNP conformers have been shown in Table 5. The notable point is a large SHG-hyperpolarizability of title compound in nonpolar medium.

Table 5

Linear and nonlinear optical parameters in different solvents and $\omega=\mathbf{0 . 0 5 1 7 7 0}$ for FDDNP conformers. Units for polarizabilities are esu $\left(\times 10^{-24}\right)$ and those for hyperpolarizabilities are esu $\left(\times 10^{-33}\right)$, calculated at B3LYP/6-31G(d,p) level of theory.

\begin{tabular}{llll}
\hline Solvent & $\begin{array}{l}\text { Linear and nonlinear optical } \\
\text { parameters }\end{array}$ & Syn & \\
\hline \multirow{3}{*}{ Water } & $\alpha(-\omega, \omega)$ & 47.85 & 46.38 \\
& $\beta(-\omega, \omega, 0)$ & 344924.10 & 320231.48 \\
& $\beta(-2 \omega, \omega, \omega)$ & 3153103.78 & 2450425.24 \\
DMSO & $\alpha(-\omega, \omega)$ & 48.86 & 47.42 \\
& $\beta(-\omega, \omega, 0)$ & 359445.29 & 333351.67 \\
& $\beta(-2 \omega, \omega, \omega)$ & 2965299.81 & 2424596.108 \\
Benzene & $\alpha(-\omega, \omega)$ & 48.40 & 47.06 \\
& $\beta(-\omega, \omega, 0)$ & 262042.98 & 244359.59 \\
& $\beta(-2 \omega, \omega, \omega)$ & 11873736.67 & 8434946.61 \\
\hline
\end{tabular}

\section{Two-state model}

As shown from Table 5, the static first hyperpolarizabilities of Syn form are larger than the similar values in Anti form. A two-state model has been employed to explain these differences. According to this model, the first hyperpolarizability can be explained using the following relation [10]:

$$
\beta \propto \Delta \mu_{g e} f_{o s} / \Delta E^{3}
$$

In the above expression, $\beta$ is proportional to the difference of dipole moment in the ground state and the crucial excited states $\left(\Delta \mu_{g e}\right)$ and the oscillator strength $\left(f_{o s}\right)$, but inversely proportional to the third power of the transition energy $(\Delta E)$. Calculated parameters by using TD-DFT method (Table 6) for FDDNP conformers indicate that the largest dipole moment changes and oscillator strengths correspond to the HOMO $\rightarrow$ LUMO transition. The transition energies and corresponding oscillator strengths are almost the same for both conformers. The difference can be attributed to a larger dipole moment change in $S y n$ form. The transition has $p \rightarrow \pi^{*}$ character (Figure 3).

Table 6

Results of the TD-DFT calculations for FDDNP conformers*.

\begin{tabular}{lllllll}
\hline & $\lambda_{\max }$ & $\Delta E$ & $f_{o s}$ & $\Delta \mu_{g e}$ & $\Delta \mu_{g e} f_{o s} / \Delta E^{3}$ & major contribution \\
\hline Syn & 416.83 & 2.8272 & 0.4904 & 15.8161 & 0.2947 & HOMO $\rightarrow$ LUMO $(97 \%)$ \\
& 275.82 & 4.4952 & 0.3425 & 13.9161 & 0.0524 & HOMO-2 $\rightarrow$ LUMO $(92 \%)$ \\
& 230.69 & 5.3742 & 0.2198 & 6.6065 & 0.0093 & HOMO-4 $\rightarrow$ LUMO $(56 \%)$ \\
Anti & 418.20 & 2.9633 & 0.4485 & 13.8768 & 0.2391 & HOMO $\rightarrow$ LUMO $(98 \%)$ \\
& 278.94 & 4.4435 & 0.2868 & 12.1143 & 0.0396 & HOMO-2 $\rightarrow$ LUMO $(92 \%)$ \\
& 236.51 & 5.2408 & 0.2337 & 4.8001 & 0.0078 & HOMO-3 $\rightarrow$ LUMO $(48 \%)$ \\
& 229.83 & 5.3932 & 0.2000 & 5.8542 & 0.0074 & HOMO-4 $\rightarrow$ LUMO $(56 \%)$ \\
\hline
\end{tabular}

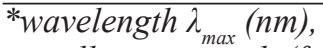

oscillator strength $\left(f_{o s} \geq 0.2\right)$,

the transition energy $\Delta E(\mathrm{eV})$,

the difference of dipole moment between the ground state and the excited state $\Delta \mu_{g e}$ (Debye).

As seen in molecular orbital plots (Figure 3), the highest occupied molecular orbitals mainly concentrate on naphthalene group and nitrogen atom in the five-member chain, while for the lowest unoccupied molecular orbitals the population analysis of molecular orbitals indicates that the C2 and C6 atoms on rotating group contribute $13 \%$ and $28 \%$ for Syn conformer and 13\% and 30\% for Anti conformer. These orbitals play the crucial role in the intramolecular chargetransfer (ICT). In this way, the electron density transfers from the electron donor moiety to the electron acceptor group. 
LUMO

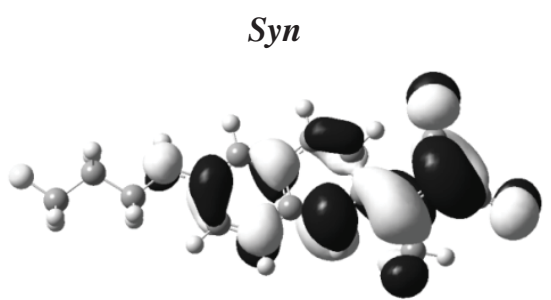

$(-2.405 \mathrm{eV})$

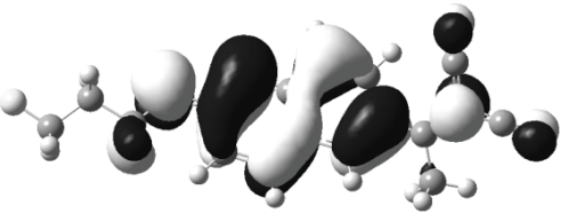

$(-5.6414 \mathrm{eV})$

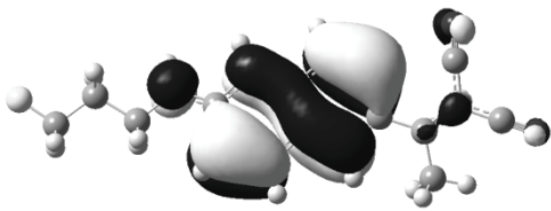

$(-6.6610 \mathrm{eV})$

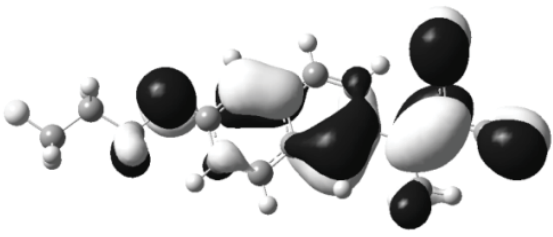

$(-7.1298 \mathrm{eV})$

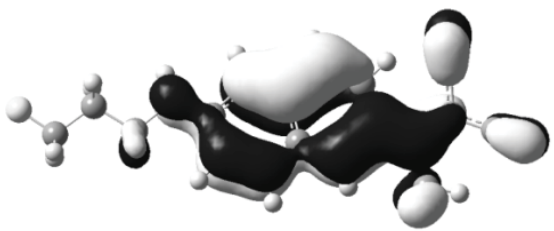

$(-8.1807 \mathrm{eV})$

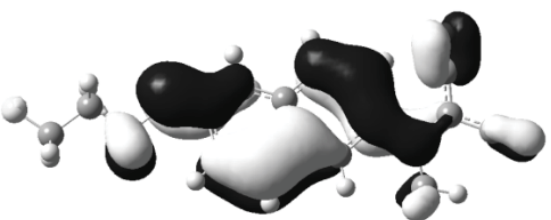

$(-8.4583 \mathrm{eV})$

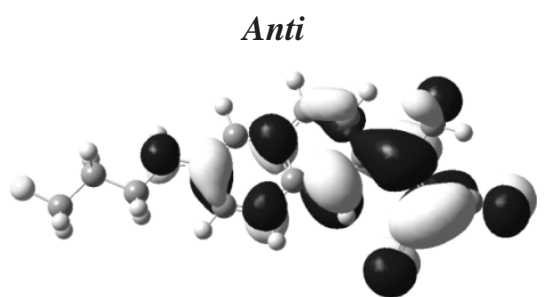

$(-2.3589 \mathrm{eV})$

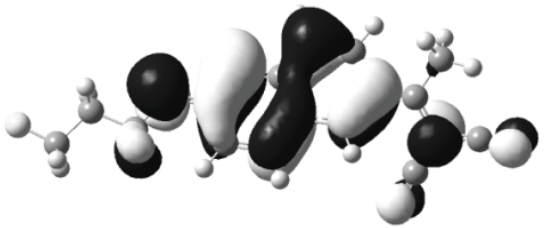

$(-5.6095 \mathrm{eV})$

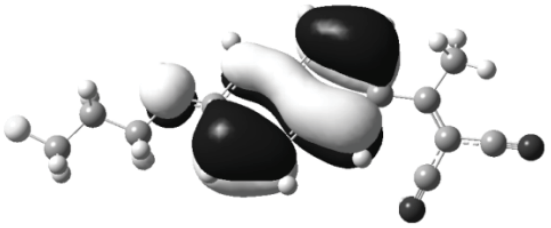

$(-6.6531 \mathrm{eV})$

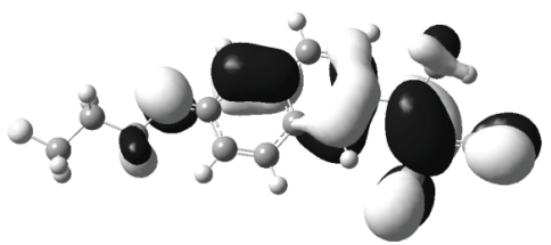

$(-7.0985 \mathrm{eV})$

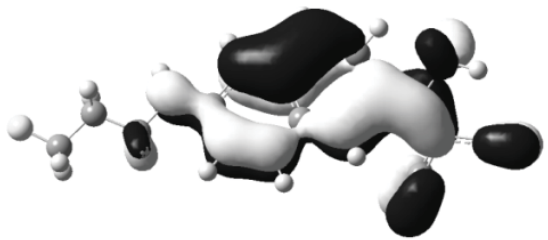

$(-8.1725 \mathrm{eV})$

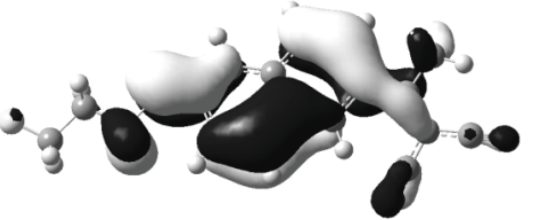

$(-8.4185 \mathrm{eV})$

Figure 3. Energy graphic representation of molecular orbitals of FDDNP conformers.

\section{Conclusions}

Two FDDNP conformers have been studied in order to predict their conformational properties and second harmonic generation potency by using density functional theory. Upon the hyperconjugation values, the Syn conformer is a more favored form. The QTAIM parameters reveal the intra-molecular hydrogen bonds in both conformers, which are classified as weak hydrogen bonds. The frequency dependent hyperpolarizabilities indicate that both conformers have high performance in near-infrared region. The best predicted working wavelength belongs to Ti:sapphire laser $(880 \mathrm{~nm})$. The conformers' first hyperpolarizability differences were explained by using the two-state model. Calculated parameters indicate that the higher first hyperpolarizability of Syn form of FDDNP is related to a larger dipole moment change in crucial excited state. The solvent effect study indicates that SHG-hyperpolarizability of FDDNP in nonpolar medium is four times greater than the similar value in polar media. 


\section{References}

1. Campagnola, P.J.; Loew, L.M. Second-harmonic imaging microscopy for visualizing biomolecular arrays in cells, tissues and organisms. Nature Biotechnology, 2003, 21, pp. 1356-1360.

2. Adur, J.; Pelegati, V.B.; de Thomaz, A.A.; Baratti, M.O.; Andrade, L.A.; Carvalho, H.F.; Bottcher-Luiz, F.; Cesar, C.L.; Cesar, C.L. Second harmonic generation microscopy as a powerful diagnostic imaging modality for human ovarian cancer. Journal of Biophotonics, 2014, 7, pp. 37-48.

3. Liu, J.; Cho, I.H.; Cui, Y.; Irudayaraj, J. Second harmonic super-resolution microscopy for quantification of mRNA at single copy sensitivity. ACS Nano, 2014, 8, pp. 12418-27.

4. Cox, G.; Kable, E.; Jones, A.; Fraser, I.; Manconi F.; Gorrell, M.D. 3-Dimensional imaging of collagen using second harmonic generation. Journal of Structural Biology, 2003, 141, pp. 53-62.

5. Jiang, J.; Yuste, R. Second-Harmonic generation imaging of membrane potential with photon counting. Microscopy and Microanalysis, 2008, 14, pp. 526-531.

6. Petric, A.; Johnson, S.A.; Pham, H.V.; Li, Y.; Ceh, S.; Golobic, A.; Agdeppa, E.D.; Timbol, G.; Liu, J.; Keum, G.; Satyamurthy, N.; Kepe, V.; Houk, K. N.; Barrio, J.R. Dicyanovinylnaphthalenes for neuroimaging of amyloids and relationships of electronic structures and geometries to binding affinities. Proceedings of the National Academy of Sciences U.S.A., 2012, 109, pp. 16492-16497.

7. Shoghi-Jadid, K.; Small, G.W.; Agdeppa, E.D.; Kepe, V.; Ercoli, L.M.; Siddarth, P.; Read, S.; Satyamurthy, N.; Petric, A.; Huang S.C.; Barrio, J.R. Localization of neurofibrillary tangles and beta-amyloid plaques in the brains of living patients with Alzheimer disease, The American Journal of Geriatric Psychiatry, 2002, 10, pp. 24-35.

8. Bresjanac, M.; Smid, L.M.; Vovko, T.D.; Petric, A.; Barrio J.R.; Popovic, M. Molecular-imaging probe 2-(1-[6-[(2-fluoroethyl)(methyl) amino]-2-naphthyl]ethylidene) malononitrile labels prion plaques in vitro. The Journal of Neuroscience, 2003, 23, pp. 8029-8033.

9. Thompson, P.W.; Ye, L.; Morgenstern, J.L.; Sue, L.; Beach, T.G.; Judd, D.J.; Shipley, N.J.; Libri, V.; Lockhart, A. Interaction of the amyloid imaging tracer FDDNP with hallmark Alzheimer's disease pathologies. Journal of Neurochemistry, 2009, 109, pp. 623-630.

10. Oudar J.L.; Chemla, D.S. Hyperpolarizabilities of the nitroanilines and their relations to the excited-state dipole moment. The Journal of Chemical Physics, 1977, 66, pp. 2664-2668.

11. Frisch, M.J.; Trucks, G.W.; Schlegel, H.B. et al. GAUSSIAN 98, (Revision A.7, Gaussian, Inc., Pittsburgh PA, 1998).

12. Lee, C.; Yang, W.; Parr, R.G. Development of the Colle-Salvetticonelation energy formula into a functional of the electron density. Physical Review B, 1988, 37, pp. 785-798.

13. Becke, A.D. Density-functional thermochemistry. III. The role of exact exchange. Journal of Chemical Physics, 1993, 98, pp. 5648-5652.

14. Stevens, P.J.; Devlin, F.J.; Chabalowski, C.F.; Frisch, M.J. Ab initio calculation of vibrational absorption and circular dichroism spectra using density functional force fields. Journal of Physical Chemistry, 1994, 98, pp. 11623-11627.

15. Hehre, W.J.; Ditchfield, R.; Pople, J.A. Self - Consistent Molecular Orbital Methods. XII. Further Extensions of Gaussian-Type Basis Sets for Use in Molecular Orbital Studies of Organic Molecules. Journal of Chemical Physics, 1972, 56, pp. 2257-2261.

16. Boyle, N.M.; Tenderholt, A.L.; Langner, K.M. A library for package independent computational chemistry algorithms. Journal of Computational Chemistry, 2008, 29, pp. 839-845.

17. Tomasi, J.; Persico, M. Molecular interactions in solution: an overview of methods based on continuous distributions of the solvent. Chemical Reviews, 1994, 94, pp. 2027-2094.

18. Lu, T.; Chen, F. Multiwfn: A multifunctional wave function analyzer. Journal of Computational Chemistry, 2012, 33 , pp. 580-592.

19. Sebastian, S.; Sylvestre, S.; Jayabharathi, J.; Ayyapan, S.; Amalanathan, M.; Oudayakumar, K.; Herman, I.A. Study on conformational stability, molecular structure, vibrational spectra, NBO, TD-DFT, HOMO and LUMO analysis of 3,5-dinitrosalicylic acid by DFT techniques. Spectrochimica Acta Part A: Molecular and Biomolecular Spectroscopy, 2015, 136, pp. 1107-1118.

20. Akhtari, K.; Hassanzadeh, K.; Fakhraei, B.; Akhtari, G. Magnetic exchange coupling of chalcogen-centered radicals mediated via the 2D curved $\pi$-network: A broken-symmetry approach. Computational Materials Science, 120, 2016, pp. 53-59.

21. Rozas, I.; Alkorta, I.; Elguero, J. Behavior of ylides containing N, O, and C Atoms as hydrogen bond acceptors. Journal of the American Chemical Society, 2000, 122, pp. 11154-11161. 
22. Shainyan, B.A.; Chipanina, N.N.; Aksamentova, T.N.; Oznobikhina, L.P.; Rosentsveig G.N.; Rosentsveig, I.B. Intramolecular hydrogen bonds in the sulfonamide derivatives of oxamide, dithiooxamide, and biuret. FT-IR and DFT study, AIM and NBO analysis. Tetrahedron, 2010, 66, pp. 8551- 8556.

23. Koch, U.; Popelier, P.L. A characterization of C-H-O hydrogen bonds on the basis of the charge density. The Journal of Physical Chemistry, 1995, 99, pp. 9747-9754.

24. Pati, S.K.; Ramasesha, S.; Shuai, Z.; Bredas, J.L. Dynamical nonlinear optical coefficients from the symmetrized density-matrix renormalization-group method. Physical Review B, 1999, 59, pp. 14827-14830.

25. Akhtari, K.; Hassanzadeh, K.; Fakhraei, B.; Hassanzadeh, H.; Akhtari, G.; Zarei, S.A. First hyperpolarizability orientation in [70]PCBM isomers: A DFT study. Computational and Theoretical Chemistry, 2014, 1038, pp. 1-5.

26. Librando, V.; Alparone, A.; Minniti, Z. Computational study on dipole moment, polarizability and second hyperpolarizability of nitronaphthalenes. Journal of Molecular Structure: THEOCHEM. 2008, 856, pp. 105-111.

27. Heisterkamp, A.; Maxwell, I.Z.; Mazur, E.; Underwood, J.M.; Nickerson, J.A.; Kumar, S. Pulse energy dependence of subcellular dissection by femtosecond laser pulses. Optics Express, 2005, 13, pp. 3690-3696.

28. Vogel A.; Venugopalan, V. Mechanisms of pulsed laser ablation of biological tissues. Chemical Reviews, 2003, 103, pp. 577-644.

29. Hassanzadeh, K.; Akhtari, K.; Hassanzadeh, H.; Zarei, S.A.; Fakhraei, N.; Hassanzadeh, K. The role of structural $\mathrm{C}-\mathrm{H}$ single bond compared with phenolic $\mathrm{OH}$ sites on the antioxidant activity of oleuropein and its derivatives as a great non-flavonoid family of the olive components: A DFT study. Food chemistry, 2014, 164, pp. 251-258.

30. Markovic, Z.; Milenkovic, Đ.; Đorovic, J.; Dimitric Markovic, J.M.; Stepanic, V.; Lucic B.; Amic, D. PM6 and DFT study of free radical scavenging activity of morin. Food chemistry, 2012, 134, pp. 1754-1760.

31. Akhtari, K.; Hassanzadeh, K.; Fakhraei, B.; Fakhraei, N.; Hassanzadeh, H.; Akhtari, G.; Zarei, S.A.; Hassanzadeh, K. Mechanisms of the hydroxyl and superoxide anion radical scavenging activity and protective effect on lipid peroxidation of thymoquinone: A DFT study. Monatsheftefür Chemie, 2015, 146, pp. 601-611. 